\title{
Discrete Multiobjective Optimization Methodology applied to the Mixed Actuators Problem and Tested in a Hardware-in-the-loop Rendezvous Simulator
}

\author{
W. Gomes dos Santos * and E. Marconi Rocco ${ }^{\dagger}$ \\ National Institute for Space Research (INPE), São José dos Campos, SP, 12227010, Brazil
}

T. Boge ${ }^{\ddagger}$, F. Rems ${ }^{\S}$ and H. Benninghoff $₫$

German Aerospace Center (DLR), Wessling, Bayern, 82234, Germany

\begin{abstract}
The spacecraft control problem using a set of actuators with conflicting characteristics is investigated in this paper. A novel approach, called actuator multiobjective command method, based on a discrete multiobjective optimization technique is proposed. The method is included in a coupled translational and attitude control system applied to the final approach rendezvous. Furthermore, all elements of the guidance, navigation and control loop have been developed and implemented in a simulation framework. A reaction control system, a set of reaction wheels, and a set of magnetic torqrods are the group of actuators used in this work. The discrete multiobjective problem is formulated with four objectives: torque error, fuel and electrical charge consumption, disturbance of coupling, and risk of utilization. The decision variable represent the command torque to the actuators. In addition, the hardware-in-the-loop rendezvous and docking simulation facility of the German Aerospace Center has been used to test the proposed method under realtime conditions. Results indicate that a mixed actuators methodology can achieve better performance with respect to those using the same type of actuators.
\end{abstract}

\section{Nomenclature}

$\mathbf{u}(h) \quad$ Discrete control signal

$\mathbf{T}_{c} \quad$ Controller torque command, Nm

$\dot{\mathbf{h}}_{w} \quad$ Reaction wheel control torque, $\mathrm{Nm}$

$\mathbf{T}_{m} \quad$ Magnetic control torque, $\mathrm{Nm}$

$\mathbf{T}_{r} \quad$ Control torque of the reaction control system, Nm

$\mathbf{T}_{d b} \quad$ Disturbance torque, Nm

$\mathrm{x}$ Commanded torque (discrete decision variable), $\mathrm{Nm}$

$\mathbf{x}^{b} \quad$ Best compromise solution, $\mathrm{Nm}$

$\mathbf{x}^{*} \quad$ Ideal solution, $\mathrm{Nm}$

p Combinatorial vector

$\mathbf{Z}(\mathbf{x}) \quad$ Objective functions vector

Subscript

$r \quad$ Reaction control system

$w \quad$ Reaction wheel

$m \quad$ Magnetic torqrod

\footnotetext{
*PhD. Student, Space Engineering and Technology, Av. dos Astronautas 1758, willer.gomes@inpe.br.

${ }^{\dagger}$ Professor, Space Engineering and Technology, Av. dos Astronautas 1758.

¥Project leader, Spaceflight Technology, Münchner Strasse 20.

$\S$ Member of Scientific Staff, Spaceflight Technology, Münchner Strasse 20.

『Member of Scientific Staff, Spaceflight Technology, Münchner Strasse 20.
} 


\section{Introduction}

$\mathrm{T}$ HE performance and robustness of an attitude and orbit control subsystem (AOCS) are highly dependent on the spacecraft actuators, such as thrusters, reaction wheels, and magnetic torqrods. Those actuators are designed to provide force and/or torque throughout the spacecraft's life in order to counteract orbital disturbances and to perform attitude and orbital maneuvers. Thrusters can be used as reaction control system (RCS) providing force and torque simultaneously, whereas reaction wheels and magnetic torqrods are on-board actuators used only for attitude maneuvers. In rendezvous and docking maneuvers, specially during the final approach phase, AOCS plays an important role mainly because the six degrees of freedom of motion must be controlled simultaneously. ${ }^{1}$

In the recent literature, hybrid controllers with mixed actuators have been the subject of extensive study. Ref. 2 compares several control methods for attitude control of a satellite with thrusters, magnetic torqrods and reaction wheels. Tracking control laws for a control system with three momentum wheels and a set of thrusters are implemented in Ref. 3. Reaction wheels failures in the Far Ultraviolet Spectroscopic Explorer satellite prompted modifications of the three-axis attitude-control so that to restore its functionality using a hybrid controller with magnetic torqrods and reaction wheels. ${ }^{4}$ A mixed actuator mode with reaction wheels and thrusters has also been uploaded to the spacecraft Dawn in early 2011. ${ }^{5}$ Recently, Ref. 6 have evaluated the feasibility of using two reaction wheels and a set of thrusters in a contingency scenario in order to meet the Cassini spacecraft pointing requirements. Their results indicated that better attitude-control performance can be achieve with hybrid controllers. In order to optimize the performance of the AOCS, many researches have applied multiobjective optimization approaches as alternative control techniques for complex nonlinear systems. ${ }^{7-9}$ A survey of multiobjective optimization methods for application in engineering has been presented in Ref. 10.

This paper introduces the problem of how to optimally command a group of distinct actuators included in a coupled complex nonlinear control system, such as that used in the final approach phase of a rendezvous maneuver. The motivation for this work is based on the autonomous operation of on-orbit servicing missions - a service satellite approaches a client satellite for inspection and capture - which has been received great attention by the space agencies recently. ${ }^{11-13}$

A novel approach to solve the mixed actuator problem is introduced based on a discrete multiobjective optimization methodology. The purposed method manages a set of thrusters, reaction wheels, and magnetic torqrods during a final approach rendezvous process taking into account the coupling between the rendezvous and attitude closed-loop control systems. In addition, models for all components of the entire guidance, navigation, and control (GNC) loop have been developed and implemented in a simulation framework. The optimization results are affected by the limitations of the actuators. Therefore, the actuators must be modeled very precisely, including all nonlinearities and saturation levels. The multiobjective problem is formulated with four conflicting objectives. The commanded torque for each actuator is treated as the decision variable. Furthermore, the proposed methodology has been integrated and tested in the hardwarein-the-loop (HIL) rendezvous and docking simulation facility of the German Aerospace Center (DLR), called European Proximity Operations Simulator (EPOS). ${ }^{14}$ In recent years, many relevant experiments have been done at this facility which uses two industrial robots to physically simulate the complete translational and rotational motion of two docking satellites. ${ }^{15,16}$ At EPOS, the entire GNC loop has been tested under realistic environmental conditions using rendezvous sensor-hardware. Moreover, this paper presents results derived by purely software simulations as well as generated by HIL-tests.

This paper is organized as follows: in Section II, a complete description of the GNC loop components is presented; the discrete multiobjective optimization problem and the proposed methodology are formulated in Section III; Section IV offers the relevant results in purely software and HIL-test simulations as well as an overview about the EPOS facility and the test setup, whereas the conclusion is drawn in Section V.

\section{Modeling of the GNC Loop}

The relative motion of the final approach rendezvous process of two satellites is implemented in numerical models. In this process, the active vehicle (chaser) is led into the the vicinity of, and eventually into contact with, the passive vehicle (target). The relative position and velocity are calculated in the Clohessy-Wiltshire coordinate frame ${ }^{1}$ whose origin is located at the center of mass (CoM) of the target spacecraft. The docking capture conditions are achieved through the V-bar axis using a closed-loop controlled straight line trajectory. 
The attitude motion is described by the body coordinate frame with the origin in the CoM of the chaser. A complete block diagram of the coupled rendezvous and attitude GNC loop is depicted in figure 1.

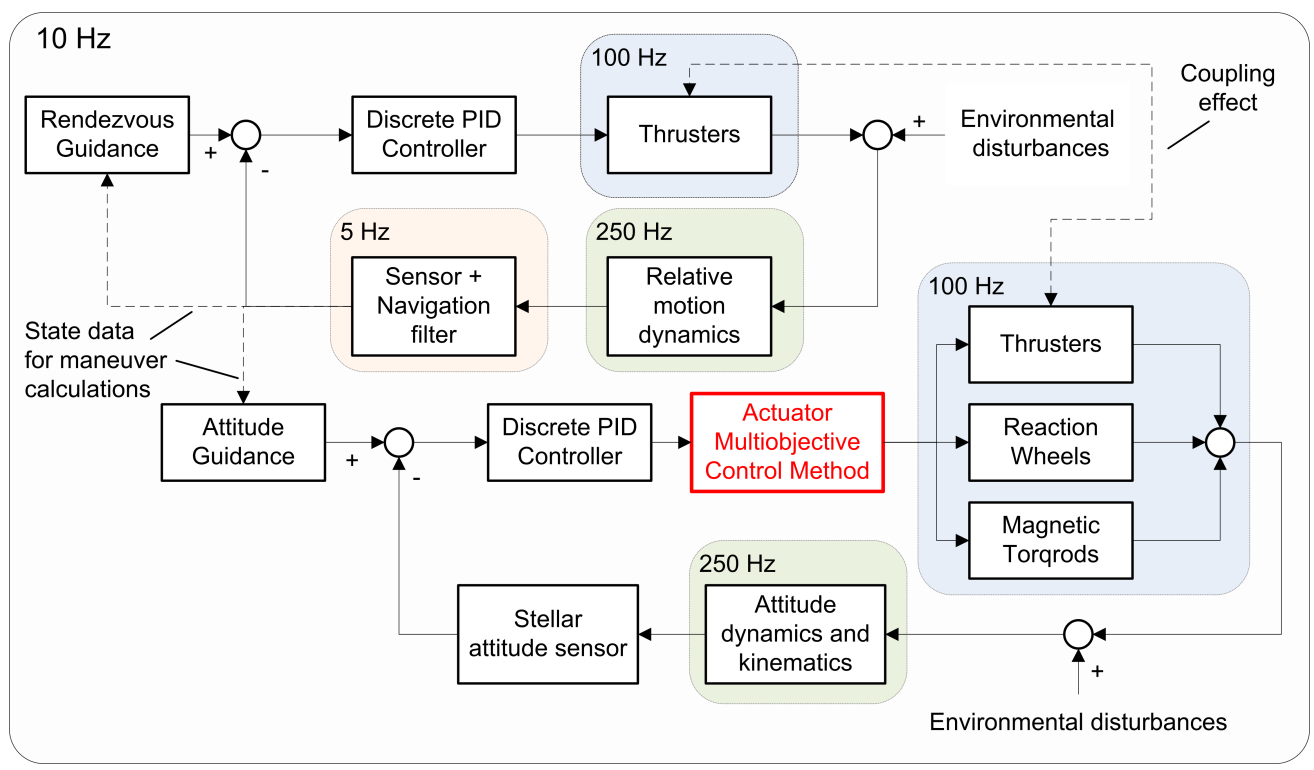

Figure 1. Block diagram of the coupled rendezvous and attitude GNC loop.

The chaser's GNC system has to act so that the relative position and velocity become zero and it has to simultaneously synchronize the attitude of both spacecraft. The translational motion is controlled via thrusters whereas the attitude control system can use thrusters, reaction wheels and/or magnetic torqrods. As shown in figure 1, the GNC loop has multiple sample times: the actuators models operate with a frequency of $100 \mathrm{~Hz}$; the relative pose estimation is provided with an update rate of $5 \mathrm{~Hz}$; the controller, guidance, and attitude sensor models work with $10 \mathrm{~Hz}$, and the dynamics models execute with a frequency of $250 \mathrm{~Hz}$. The chaser's relative translational motion, in the target centered local orbital frame, is described by Hill equations. ${ }^{1}$ The nonlinear rotational dynamics of the spacecraft is expressed by Euler's equations whereas the attitude kinematics is expressed by Euler angles and quaternions. ${ }^{17}$ The 3-2-1 attitude transformation is used in this paper. The kinematic equations and the Euler's equations of rotational dynamics complete the set of differential equations needed to describe the change in attitude of a rigid body under the influence of a time varying torque.

Two types of external disturbances have been considered in this work: residual atmospheric drag ${ }^{1,18}$ and gravity gradient moment. ${ }^{19}$ Both disturbances play an important role in low Earth orbit (LEO). They are continuously counteracted by the control systems. The guidance subsystems provide reference values at each sample time generating position and attitude profiles for the respective control system. Thereby, the reference values are compared with the estimated values, provided by the navigation subsystem, in order to compute the control commands by the control subsystems. Regarding the rendezvous guidance subsystem, an acceleration profile has been implemented in order to reach the desired approach velocity. The rendezvous guidance profile consists of an acceleration phase, a constant velocity phase, and a deceleration phase. ${ }^{1}$ For rotational motion, the attitude guidance subsystem calculates over time the necessary rotation to keep the chaser body frame aligned with the target docking axis.

The chaser relative position and attitude are measured by a vision sensor and smoothed by a navigation filter. The extended Kalman filter ${ }^{20}$ is used to estimate the relative position. This filter is an estimator with real-time characteristics, i.e., it tries to minimize the variance of the estimation error at each sample time. The estimated chaser state is also used by the guidance functions to compute the reference states. A mathematical model ${ }^{21}$ of the vision camera CCD sensor is used in purely software simulations whereas the corresponding real sensor is employed in real-time HIL simulations. The magnitude of the expected error of the relative position is pre-estimated based on geometrical projection of the target on the camera CCD sensor, i.e., the measurements errors vary with the approaching distance. The chaser's absolute attitude is provided by an accurate stellar attitude sensor with an update rate of $10 \mathrm{~Hz}$. Noise and bias are assumed in both sensor models. 
The objective of the control function is to calculate the force and torque commands needed to be applied to the spacecraft to correct the deviations in the state. Here a discrete proportional-integral-derivative (PID) controller has been designed in order to ensure the stability of the GNC loop, to keep low steady state errors, and to have a good noise rejection capability. The discrete control signal is given $a^{22}$

$$
u(h)=K_{p} e(h)+\left[I\left(h+\Delta h_{c}\right)-\frac{K_{p} \Delta h_{c}}{T_{i}} e(h)\right] S_{w}+K_{p} T_{d} \dot{e}(h)
$$

where $e(h)$ and $\dot{e}(h)$ are the position and velocity errors, respectively, between the desired and the estimated state; $K_{p}$ is the proportional gain; $T_{i}$ is the integral time constant; $T_{d}$ is the derivative time constant; $\Delta h_{c}$ is the controller sample period; $I\left(h+\Delta h_{c}\right)$ is the integral control signal; and the parameter $S_{w}$ switches the integral action in order to avoid the integral windup effect. ${ }^{23}$ This phenomenon is usually created by nonlinear actuators. If the actuator output is in the saturation region then the control signal has no effect on the actuator output. The practical consequence of this behavior is the reversing of the process to open-loop control or the exhibition of excessive overshoot in the process output.

In many works, actuators are assumed as ideal, i.e., the control signal is applied directly to the controlled process. However, it is evident that the actuator dynamics, as the nonlinear behavior of thrusters or of electromechanical devices, can affect the performance of the control system. Considerable effort has been made to model the complex and nonlinear dynamics behavior of actuators and its impact on spacecraft control systems in order to meet the increasing requirements of space applications. ${ }^{24,25}$ The following subsections describe the mathematical modeling of the actuators used in this work.

\section{A. Reaction Control System}

A set of fixed small thrusters of constant thrust allow the control of translational and rotational motion of the spacecraft on all axes. In order to have a varying amplitude thruster control, RCS is used in a quasi-linear mode by applying the pulse width pulse frequency (PWPF) modulation. ${ }^{19}$ The pulse length can vary from the minimum impulse bit (MIB) - lower pulse duration of a delivered thrust - to the control sample time. In this work, the RCS is composed of 12 one-sided thrusters. Figure 2 illustrate the locations and orientations of the thrusters.
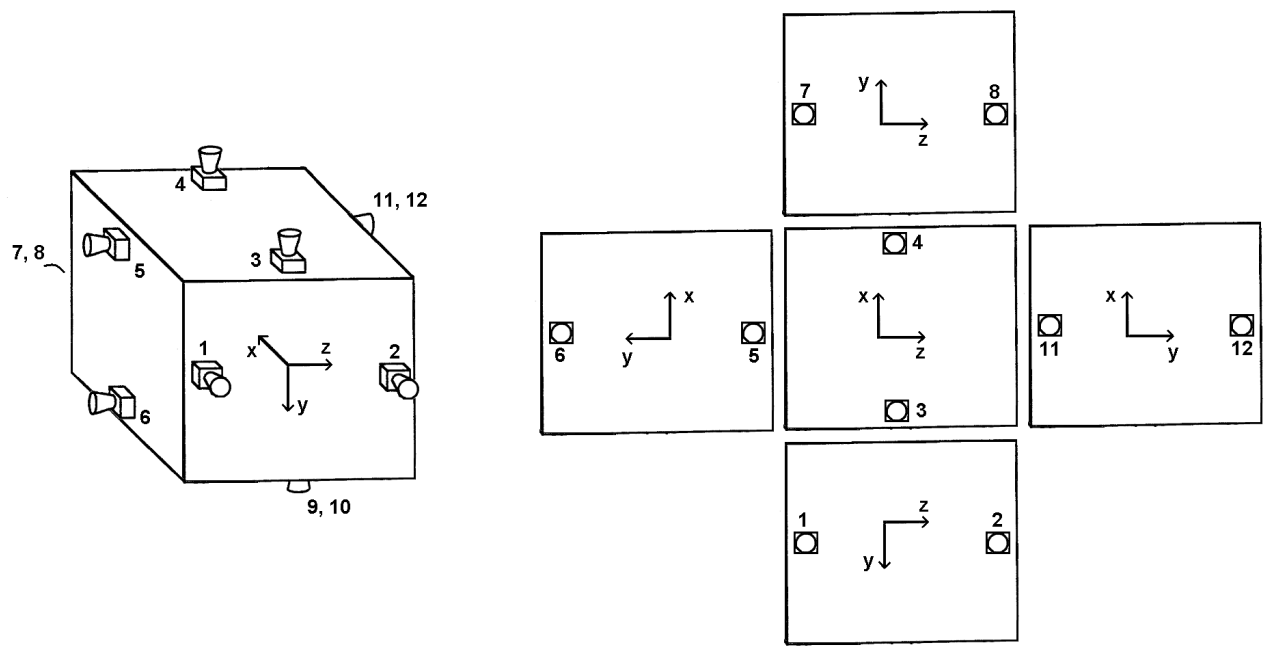

Figure 2. Thrusters' location and orientation for the assumed satellite.

The force and torque provided by the thrusters can be expressed as ${ }^{1}$

$$
\mathbf{F}=\mathbf{A} \mathbf{u}_{t}
$$

where $\mathbf{F} \in \mathbb{R}^{6}$ is the requested control vector composed of three components of force and torque respectively; if $n$ is the number of thrusters, then $\mathbf{u}_{t} \in \mathbb{R}^{n}$ is a normalized vector which represents the firing pulse duration commanded to the thruster; and $\mathbf{A} \in \mathbb{R}^{6 \times n}$ is the configuration matrix which defines the positions 
and orientations of the thrusters in the vehicle body frame. The configuration matrix, composed of the maximum thrust level $f$ and the maximum achievable torque $f r$ is given by

$$
\mathbf{A}=\left[\begin{array}{cccccccccccc}
f & f & 0 & 0 & 0 & 0 & -f & -f & 0 & 0 & 0 & 0 \\
0 & 0 & f & f & 0 & 0 & 0 & 0 & -f & -f & 0 & 0 \\
0 & 0 & 0 & 0 & f & f & 0 & 0 & 0 & 0 & -f & -f \\
0 & 0 & 0 & 0 & f r & -f r & 0 & 0 & 0 & 0 & -f r & f r \\
f r & -f r & 0 & 0 & 0 & 0 & -f r & f r & 0 & 0 & 0 & 0 \\
0 & 0 & f r & -f r & 0 & 0 & 0 & 0 & -f r & f r & 0 & 0
\end{array}\right]
$$

where $r$ represents the distance of the $i$ th thruster from the CoM of the body vehicle.

The coupling problem is treated using a flexible approach ${ }^{26}$ where the optimal set of thrusters and the firing command duration are defined on-board by a real-time algorithm. The solution of the thruster allocation problem, expressed by Eq. 2, involves solving a linear optimization problem ${ }^{27}$ for the variable $\mathbf{u}_{t}$, given $\mathbf{F}$ and $\mathbf{A}$, with the linear constraint of $\mathbf{u}_{t} \geqslant 0$. So the thruster management function computes at every control cycle the best combination of thrusters and the firing duration commands that meet the force and torque required by the controller. When the magnitude of the commanded outputs cannot be accomplished, then the vector $\mathbf{u}_{t}$ is computed in order to achieve at least the requested directions. In addition to the rounding and quantization errors, random errors, bias, and delays can also be considered in the modeling.

An important parameter that plays a great role in the design of RCS is the type of propulsion system. Here, cold gas is assumed to be the stored propellant. The basic equation of propellant mass consumption $m_{p}(t)$ is defined $\operatorname{as}^{28}$

$$
m_{p}(t)=\int_{t_{0}}^{t} \frac{f(\tau)}{I_{s p} g_{0}} d \tau
$$

where $g_{0}$ is the Earth gravitational constant; and $I_{s p}$ is the specific impulse of the propellant. When very short pulses (near the MIB) are activated, the specific impulse is significantly reduced, increasing the propellant consumption consequently. ${ }^{19}$

\section{B. Reaction Wheels}

In an attitude control system, reaction wheels are able to provide very accurate response for reasonably fast maneuvers through the principle of conservation of angular momentum. The reaction torque is realized from the response of a flywheel whose rate of velocity change is proportional to its output. An electric motor drives the flywheel rotation implying in a variable adjustment of torque. The error between the commanded and the applied torque is mainly caused by nonlinear friction disturbances inherent to the nonlinear physical characteristics of the electric motor. ${ }^{29}$ It is worthwhile to mention that such disturbances are also responsible for increasing the electric charge consumption. The dynamic model of an electromechanical motor is used to represent the reaction wheel, ${ }^{19}$ as shown in figure 3. Furthermore, it is assumed a physical set-up of three identical reaction wheels whose axes of rotation are aligned with the body axes frame.

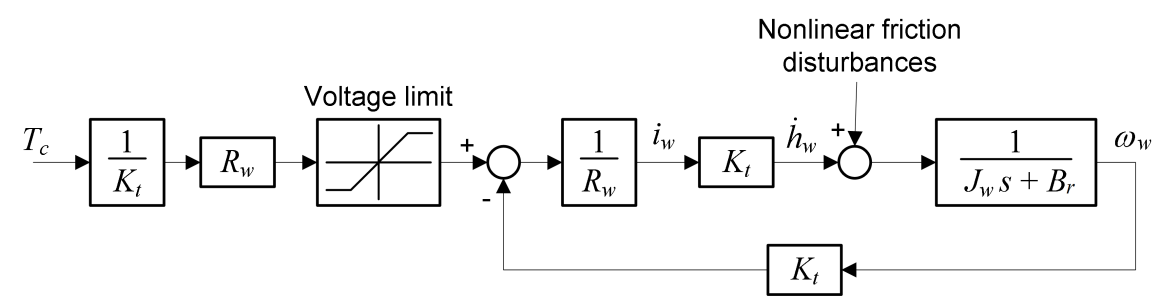

Figure 3. Electromechanical model of a reaction wheel.

In figure $3, T_{c}$ is the torque command of the controller; $K_{t}$ is the flywheel torque coefficient; $R_{w}$ is the electrical resistance of the motor armature; $i_{w}$ is the motor current; $\dot{h}_{w}$ is the achieved inertial torque; $\omega_{w}$ is the flywheel angular velocity; $J_{w}$ is the flywheel moment of inertia; and $B_{r}$ is the viscous damping coefficient of the rotor. 


\section{Magnetic torqrods}

A reliable and cost efficient approach of attitude control is the use of magnetic torqrods, specially for LEO satellites. The control torque is generated through the interaction between the on-board electromagnetic dipole moment and the geomagnetic field. Such dipole moment is induced by a set of three orthogonal current-driven coils. The major disadvantage of these devices is that the applied torque is constrained in a plane perpendicular to the Earth's magnetic field vector and depends on the strength of it as well. ${ }^{30} \mathrm{In}$ particular, it turns out that it is not possible to control all three spacecraft axes using only magnetic actuators. Hence, at least, one reaction wheel is usually used in cooperation with magnetic torqrods. Nevertheless, the variability of the geomagnetic field along the spacecraft orbit plays an important role for the behavior of such actuators ensuring the controllability of the attitude dynamics. The most accepted model for Earth's magnetic field is the International Geomagnetic Reference Field ${ }^{31}$ developed by the International Association of Geomagnetism and Aeronomy.

The magnetic control policy is based on the principle of perpendicularity ${ }^{19,32}$ which takes into account only the component of the commanded torque vector $\left(\mathbf{T}_{c} \in \mathbb{R}^{3}\right)$ perpendicular to the geomagnetic field vector $\left(\mathbf{b} \in \mathbb{R}^{3}\right)$. Then the magnetic control torque $\left(\mathbf{T}_{m} \in \mathbb{R}^{3}\right)$ is expressed by

$$
\mathbf{T}_{m}=\mathbf{B m}=\frac{1}{|\mathbf{b}|^{2}} \mathbf{B B}^{\prime} \mathbf{T}_{c}
$$

where $\mathbf{m} \in \mathbb{R}^{3}$ is the magnetic dipole moment; and the matrix $\mathbf{B} \in \mathbb{R}^{3 \times 3}$ is composed of the components of the Earth's magnetic field in the body reference frame, such as

$$
\mathbf{B}=\left[\begin{array}{ccc}
0 & b_{z} & -b_{y} \\
-b_{z} & 0 & b_{x} \\
b_{y} & -b_{x} & 0
\end{array}\right]
$$

The torqrod, composed of a magnetic coil, generates a magnetic moment when energized whose equation is describe by ${ }^{17}$

$$
\mathbf{m}=i_{m} N A_{m} \mu \hat{\mathbf{n}}
$$

where $i_{m}$ is the electrical current; $N$ is the number of coils; $A_{m}$ is the cross-sectional area of the loop; $\mu$ is the permeability of the core material; and $\hat{\mathbf{n}} \in \mathbb{R}^{3}$ is a unit vector in the direction of the magnetic coil dipole. In this paper, we assume a physical set-up of three magnetic torqrods aligned with the spacecraft principal axes.

Therefore, the Euler's dynamic equation of a satellite attitude motion composed of reaction thrusters, reaction wheels and magnetic torqrods can be described as ${ }^{19}$

$$
\mathbf{J} \dot{\boldsymbol{\omega}}=\mathbf{T}_{m}+\mathbf{T}_{r}+\mathbf{T}_{d b}-\dot{\mathbf{h}}_{w}-\boldsymbol{\omega} \times\left(\mathbf{J} \boldsymbol{\omega}+\mathbf{J}_{w} \boldsymbol{\omega}_{w}\right)
$$

where $\mathbf{J} \in \mathbb{R}^{3}$ is the moment of inertia vector of the satellite; $\boldsymbol{\omega} \in \mathbb{R}^{3}$ is the angular velocity vector of the satellite body relative to the inertial coordinate frame; $\mathbf{T}_{r} \in \mathbb{R}^{3}$ is the torque vector applied by the RCS; and $\mathbf{T}_{d b} \in \mathbb{R}^{3}$ is the overall disturbance torque vector.

\section{The Actuator Multiobjective Command Method}

Multiobjective optimization approaches have begun to be used mainly in areas of economy and operational research. Nowadays, its application to several real-world problems has received much more attention. This technique pursues to solve problems of conflicting objectives in a systematical and simultaneous way. Different approaches have been developed to solve multiobjective problems in the engineering field. ${ }^{10}$ Here, a novel discrete multiobjective optimization methodology, called Actuator Multiobjective Command Method (AMCM), is developed and used to solve the mixed actuator problem. The AMCM is responsible for commanding the necessary torque for the actuators at every control cycle based on the requested torque level and the current situation of the actuators dynamics.

According to Ref. 33, the general multiobjective optimization problem is stated as follows

$$
\begin{aligned}
& \text { Minimize } \mathbf{Z}(\mathbf{x})=\left[Z_{1}(\mathbf{x}), Z_{2}(\mathbf{x}), \ldots, Z_{p}(\mathbf{x})\right] \\
& \text { subject to } \mathbf{x} \in \mathbf{X}
\end{aligned}
$$


where the decision space $\mathbf{X} \in R^{k}$ (for $k \geq 1$ ) is a given finite feasible set; $\mathbf{x} \in \mathbb{R}^{n}$ is a vector of $n$ decision variables; $p$ is the number of objective functions; and $\mathbf{Z}(\mathbf{x}) \in \mathbb{R}^{p}$ is the vector of objective functions. The optimal solution which minimizes the objective function vector $\mathbf{Z}(\mathbf{x})$ is denoted by $\mathbf{x}^{*}$. Discrete problems are characterized by a predefined set of alternatives available for the decision variables. ${ }^{34}$

In this paper, the discrete multiobjective problem is formulated with four objectives functions: torque error, fuel and electrical consumption, disturbance of coupling, and risk of utilization. The first objective function, torque error, measures the difference among the requested torque and the total applied torque. The fuel and electrical charge consumption represents the total amount of propellant mass and electrical charge consumed by the set of actuators. Due to the actuator's dynamics some disturbances can be generated in the axes different than the axis of applied torque, such behavior is denoted here by disturbance of coupling. The last objective function, risk of utilization, takes into account the failure probability of each actuator. All objectives functions are evaluated at every control cycle and they measure, in a general way, the cost and efficiency of the actuators. The discrete decision variable vector, $\mathbf{x}$, represents combinatorial combinations of the torque control signal. The three components of the decision variable vector $\left(x_{1}, x_{2}, x_{3}\right)$ have the function of commanding the actuators: reaction thruster, reaction wheel, and magnetic torqrod, respectively.

The mathematical formulation of the AMCM is given by

$$
\begin{aligned}
\mathbf{Z}(\mathbf{x}) & =\left[Z_{1}(\mathbf{x}), Z_{2}(\mathbf{x}), Z_{3}(\mathbf{x}), Z_{4}(\mathbf{x})\right] \\
\mathbf{x} & =\frac{\mathbf{p}}{k}\left|T_{c}\right| \\
Z_{1}\left(x_{1}, x_{2}, x_{3}\right) & =T_{r}^{a}\left(x_{1}\right)+T_{w}^{a}\left(x_{2}\right)+T_{m}^{a}\left(x_{3}\right)-\left|T_{c}\right| \\
Z_{2}\left(x_{1}, x_{2}, x_{3}\right) & =c_{1} x_{1}+c_{2} x_{2}+c_{3} x_{3} \\
Z_{3}\left(x_{1}, x_{2}, x_{3}\right) & =i_{1} x_{1}+i_{2} x_{2}+i_{3} x_{3} \\
Z_{4}\left(x_{1}, x_{2}, x_{3}\right) & =r_{1} x_{1}+r_{2} x_{2}+r_{3} x_{3} \\
\text { subject to } \quad x_{1}, x_{2}, x_{3} & \geqslant 0 \\
p_{1}+p_{2}+p_{3} & =k \\
\Rightarrow x_{1}+x_{2}+x_{3} & =\left|T_{c}\right| \\
k & =\frac{\Delta h_{c}}{\Delta h_{a}}
\end{aligned}
$$

where $\Delta h_{a}$ is the actuator sample period; and $k$ is the number of subsets.

The vector $\mathbf{p} \in \mathbb{R}^{n}$ denotes the combinatorial vector whose function is to quantize the torque command of the controller to yield the decision variables. The coefficients $c_{j}, i_{j}$ and $r_{j}$ (with $j=1,2$ and 3 ) have the meaning of weights and they are used to evaluate the following objective functions: fuel and electrical consumption, disturbance of coupling, and risk of utilization, respectively. They are defined according to the characteristics of the actuators. It is worthwhile to mention that the set of actuators is defined a priori, i.e., assuming a fixed scenario. It is not the purpose of this work to select actuators from suppliers. The focus of this paper is, given a set of actuators with conflicting characteristics, to define the best way to operate them.

The combinatorial problem can be solved using the $k$-combination with repetitions equation (also called by $k$-multicombinations). This theory allows to calculate the number of ways to sample $k$ elements from a set of $n$ elements allowing for duplicates. Hence, the number of multisubsets of size $k$ is then the number of non-negative integer solutions of the linear Diophantine equation, ${ }^{35}$ given as follows

$$
x_{1}+x_{2}+x_{3}+\ldots+x_{n}=k
$$

If a given set has $n$ elements, then the number of such $k$-multisubsets can be written as ${ }^{35}$

$$
\left(\begin{array}{c}
n+k-1 \\
k
\end{array}\right)=\frac{(n-1+k) !}{(n-1) ! k !}
$$


Note that, with respect to the problem discussed in this paper, $n$ represents the number of decision variables, i.e, the number of actuators $(n=3)$, whereas $k$ is the number of divisions of a given parameter. Since the actuators model executes 10 times faster than the control cycle, then the control cycle is split in 10 parts $(k=10)$. Therefore, the set of candidate solutions has a finite amount of 66 elements. The number of solutions could be increased in order to have more points in the objective space, but this action would increase the computational effort of the simulation.

The complete mathematical modeling of spacecraft actuators can be a cumbersome task due to its complexity and nonlinear dynamical behavior. ${ }^{24}$ In Eq. 10, $T_{r}^{a}\left(x_{1}\right), T_{w}^{a}\left(x_{2}\right)$, and $T_{m}^{a}\left(x_{3}\right)$ are the test torque theoretical functions of the reaction thruster, reaction wheel, and magnetic torqrod, respectively. Since is not possible to carry out laboratory testing, then numerical equations are used to emulate the dynamical behavior of the actuators, i.e., the actuator output is evaluated for every candidate torque of the decision variable space. The test torque theoretical functions have been formulated through independent curve fitting analysis of the actuators model and they are given by

$$
\begin{gathered}
T_{r}^{a}\left(x_{1}\right)=\left\{\begin{array}{r}
T_{r}^{\max }+\bar{w} \text { for } x_{1} \geqslant T_{r}^{\max } \\
x_{1}+\bar{w} \text { for } 0<x_{1}<T_{r}^{\max } \\
0 \text { for } x_{1}=0
\end{array}\right. \\
T_{w}^{a}\left(x_{2}\right)=\left\{\begin{array}{r}
x_{2}+w+s \text { for } x_{2} \leqslant T_{w}^{\max } \\
T_{w}^{\max }+w+s \text { for } x_{2}>T_{w}^{\max }
\end{array}\right. \\
T_{m}^{a}\left(x_{3}\right)=\left\{\begin{array}{r}
\left(a_{m} x_{3}^{2}+b_{m} x_{3}\right)+w+s \text { for } x_{3} \leqslant T_{m}^{\max } \\
T_{m}^{\text {max }}+w+s \text { for } x_{3}>T_{m}^{\max }
\end{array}\right.
\end{gathered}
$$

where the superscript ${ }^{\max }$ represents the theoretical maximum torque applied by the actuator; $w$ is the white Gaussian noises whose statistics is given by $w=N(0, Q)$, i.e., zero mean and covariance $Q ; s$ is the bias error; and $a_{m}$ and $b_{m}$ are the coefficients of a second degree polynomial that best fit the torque curve of the magnetic torqrod. The thruster's torque error is quite difficult to determine mainly due to the nonlinear behavior of this actuator. In this case, it is assumed the average torque error $\bar{w}$ of the last 100 step times applied by the RCS.

Unlike the single-objective optimization, where the optimal objective value is unique, multiobjective optimization leads to a group of incomparable optimal values with the same degree of optimality due to the conflicting behavior of the problem. A solution that cannot be improved in any of the objectives without degrading at least one of the other objectives is called non-dominated solution. Likewise, if a solution can be improved by another one, then it is called dominated solution. The definition of the set of optimal values, called of noninferior set or Pareto front, has been widely described in the literature. ${ }^{10,33,36}$ Since is not possible to order the candidate solutions, the ordinary notion of optimality should be dropped. ${ }^{33}$ However, especially in many practical applications, a single solution shall be found. Usually, many methods in the literature assume some preferences and reduce the problem to a single-objective optimization. ${ }^{10}$

An alternative to these approaches is the Smallest Loss Criterion ${ }^{37-39}$ which yields a single solution point, called best compromise solution, $\mathbf{x}^{b} \in R^{n}$, without prioritize any objective. This decision making method pursues the solution of smaller loss for all objectives, mathematically it relies on finding the barycenter which represents the intermediate ideal value at the objective space - of a normalized $p$-dimensional figure, where $p$ represents the number of objective functions of the problem. The normalization process is important to disregard the dimension of each objective. The method evaluates the Euclidean distance, at the objective space, from the barycenter to all candidate values. Since the closest candidate value from the barycenter is found, then a mapping to the decision space defines the best compromise solution. The function to be minimized, $\mathbf{Z}\left(\mathbf{x}^{b}\right) \in \mathbb{R}^{p}$, is given by

$$
\mathbf{Z}\left(\mathbf{x}^{b}\right)=\min \left|\mathbf{Z}(\mathbf{x})-\mathbf{Z}\left(\mathbf{x}^{*}\right)\right|=\min \left\{\sum_{1}^{p}\left[Z_{i}(\mathbf{x})-Z_{i}\left(\mathbf{x}^{*}\right)\right]^{2}\right\}^{1 / 2}
$$

where $\mathbf{Z}\left(\mathbf{x}^{*}\right) \in \mathbb{R}^{p}$ is the ideal objective value. The aforementioned explanation of the AMCM is summarized in the illustrative figure 4 . 


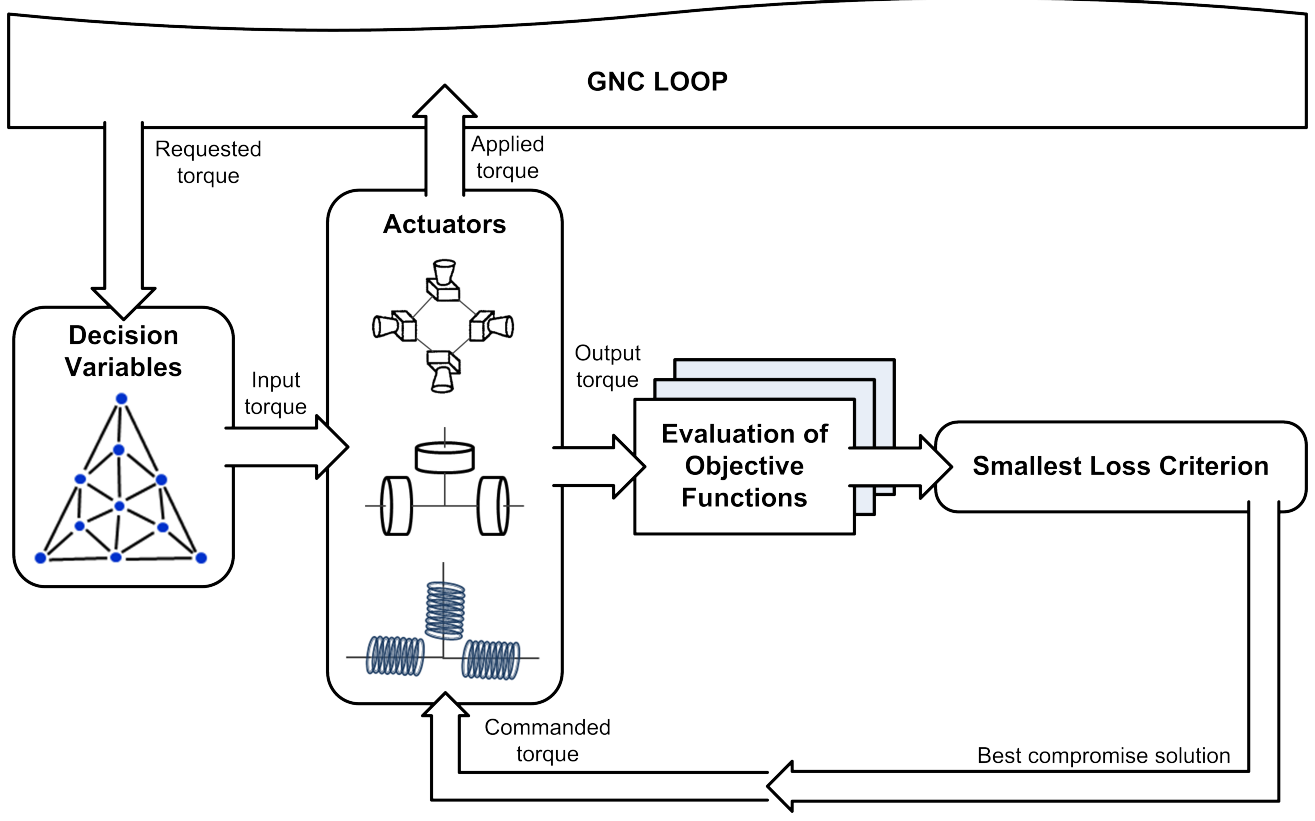

Figure 4. Functional concept of the Actuator Multiobjective Command Method.

\section{Results}

Numerical simulations of AMCM applied to the coupled rendezvous and attitude control system are presented in this section. Both non-real-time and real-time simulations have been executed. Regarding the purely numerical simulations, the proposed method is compared with the case where only the same set of actuators is used. For real-time simulations, AMCM is applied to the HIL rendezvous and docking simulator EPOS and the relevant results are shown. Table 1 introduces the main parameters of the simulations. The actuator and sensor parameters are based on data sheets provided by manufacturers.

\section{A. Discrete Multiobjective Optimization Analysis}

The AMCM evaluates the objective functions at every control cycle and selects the best compromise solution based on a discrete multiobjective optimization methodology. In this section, the RCS is composed of thrusters with nominal thrust of $0,5 \mathrm{~N}$. The combinatorial variables play an important role in this process by the quantization of the control signal. They change the decision variables, expressed by the torque commanded to the actuators, proportionally. In this paper, the combinatorial space is composed of 66 elements as shown in figure 5. The dimensionless combinatorial variable 1,2 , and 3 , are related to the decision variables: reaction thruster, reaction wheel, and magnetic torqrod, respectively. Each point in the plot provides the contribution of the actuators for meeting the requested torque.

The presentation of results is fundamental for the success of any project. However, this step is usually difficult in multiobjective analysis, where the amount of relevant information increases with the objectives. Even with few points, the three-objective graphical display may be considered awkward to understand. For more than three objectives, graphical displays on orthogonal axes must be dropped. An alternative to the typical graphical displays, adaptable to any number of objectives, was proposed in Ref. 40: the value path plot. The display consists of a set of parallel scales (one representing each objective) on which is drawn a line (a value path) that connects the objective function values between the scales. This representation allows considering simultaneously a large number of objectives. Figure 6 shows the value path representation of AMCM for two particular requested torque levels: $0.015 \mathrm{Nm}$ and $0.085 \mathrm{Nm}$.

The vertical axes represent the normalized scale of the objective functions, whose labels are shown in the horizontal axis. Every line represents a candidate solution. The optimal solution of each objective, also called primary path, is represented by a thick line: the red line optimizes $Z_{1}$, the green line optimizes $Z_{2}$, the dark blue line optimizes $Z_{3}$, and the purple line optimizes $Z_{4}$. The best compromise solution is represented 
Table 1. Simulation parameters.

\begin{tabular}{|c|c|c|c|}
\hline Orbit parameters & Value & Multiobjective coefficients & Value \\
\hline Altitude, km & 450 & Fuel and electrical consumption $(c)$ & {$[3,2,1]$} \\
\hline Eccentricity & 0 & Disturbance of coupling $(i)$ & {$[2,1,3]$} \\
\hline Inclination, degrees & 45 & Risk of utilization $(r)$ & {$[1,2,3]$} \\
\hline Mean anomaly, degrees & 90 & & \\
\hline Satellite parameters & & RCS parameters & \\
\hline Moment of inertia, kg.m² & {$[100,120,80]$} & Propellant & Cold gas \\
\hline Initial mass, $\mathrm{kg}$ & 500 & Thrust, N & $0.5 / 1$ \\
\hline Arm length, m & 0.5 & $\mathrm{MIB}, \mathrm{Ns}$ & $0.05 / 0,1$ \\
\hline Stellar attitude sensor & & Reaction wheel parameters & \\
\hline Spatial random error, arcsecond & $18.9,3 \sigma$ & Wheel moment of inertia, kg.m ${ }^{2}$ & 0.0191 \\
\hline Spatial bias error, arcsecond & $10,3 \sigma$ & Maximum torque, $\mathrm{Nm}$ & 0.054 \\
\hline Camera CCD sensor & & Magnetic torqrod parameters & \\
\hline Focal length, pixels & 604 & Magnetic dipole moment, $\mathrm{Am}^{2}$ & 170 \\
\hline Resolution, pixels & $640 \times 480$ & Maximum torque, $\mathrm{Nm}$ & 0.012 \\
\hline
\end{tabular}

* Achieved with the Earth's magnetic field of the particular orbital position.
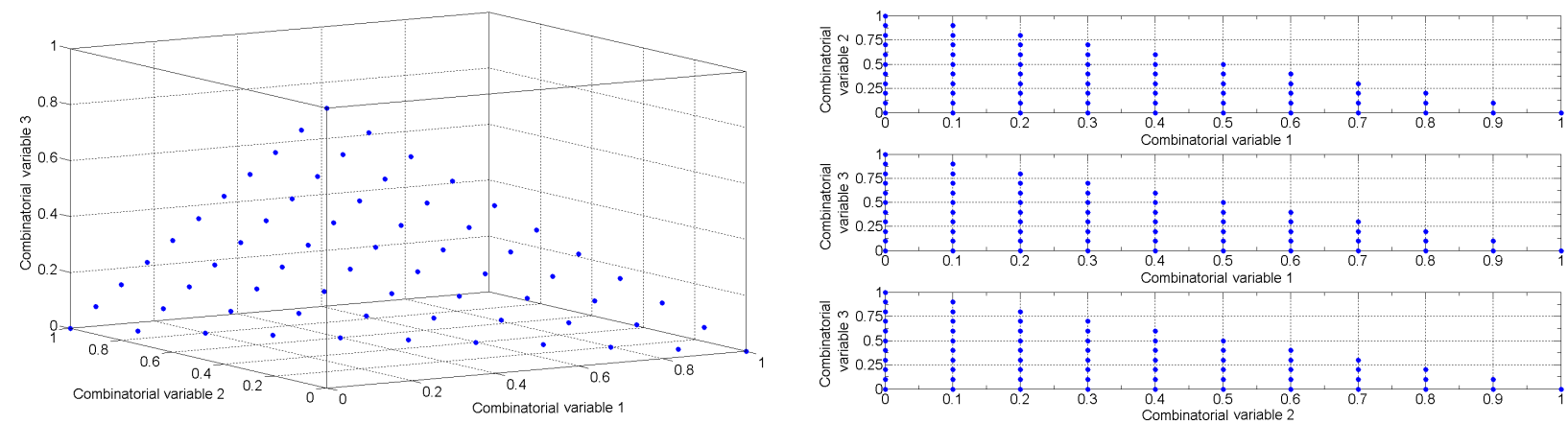

Figure 5. Combinatorial space of the discrete multiobjective problem.

by the blue dashed line. The necessary condition for noninferiority in this kind of plot is the value path must intersect all primary paths. ${ }^{40}$ In this case, all candidate solutions belong to the noninferior set. Note also that sometimes a particular solution optimizes one objective, but provides the highest values for others, e.g., the primary path of $Z_{4}$ (purple thick line) in figure 6(a).

It is worthwhile to point out that the best compromise solution is clearly an equilibrium solution among the primary paths, as defined by the smallest loss criterion. At every control cycle, new shapes of value path plots are generated. Thereupon, the torque is split according to the requested torque level, i.e., the commanded torque for each actuator changes constantly. Figure 7 shows the AMCM commanded torque (decision variables) for the best compromise solution exhibited in figure 6 .

The values represent a percentage of the total amount of the requested torque. As can be seen, in figure 7 (a) the major contribution is given by the reaction thruster and the reaction wheel with identical values (40\%), whereas in figure 7(b), since the requested torque level has increased, AMCM commanded $50 \%$ of the torque to the reaction thruster because it has a higher capacity.

So far, AMCM has been analyzed from a viewpoint of a particular control cycle. However, it makes necessary to examine also the AMCM's performance throughout the whole maneuver. Hence a final approach rendezvous simulation has been executed from $20 \mathrm{~m}$ to $10 \mathrm{~m}$. An initial angular error - difference between the actual attitude and the guidance values - of 10 degrees in the $Y_{B}$ axis and null errors for the other axes, have been assumed in this simulation. 


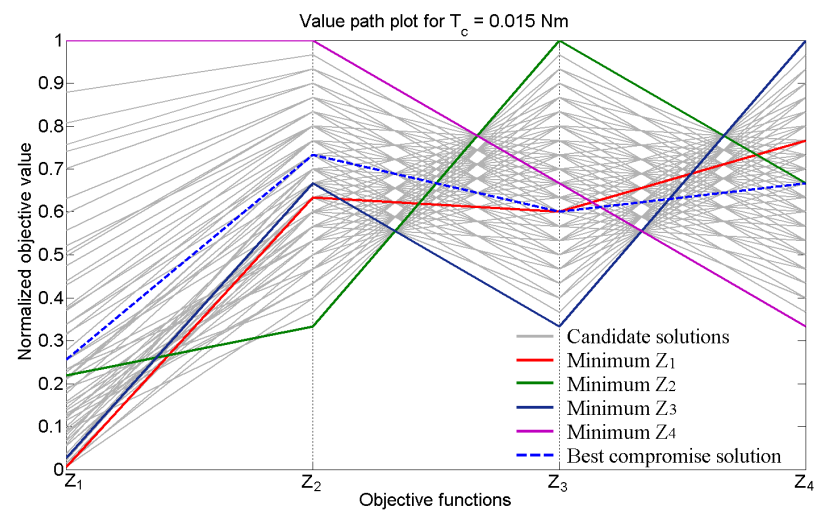

(a)

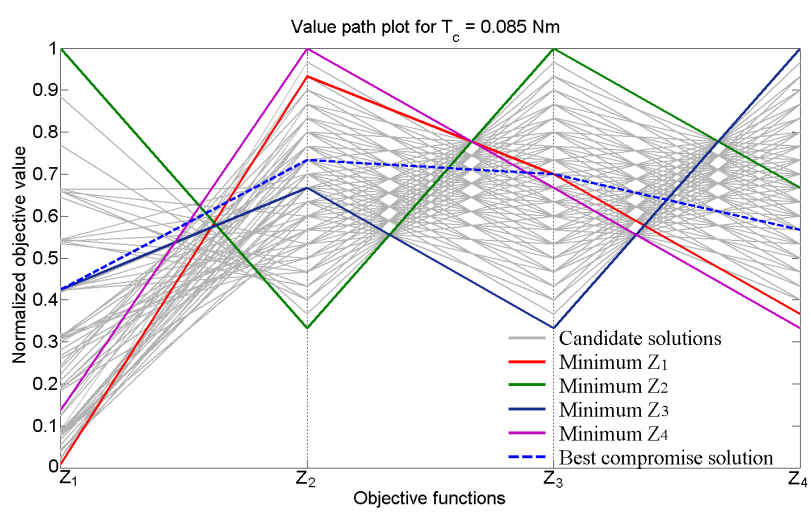

(b)

Figure 6. Value path plot of AMCM: (a) requested torque of $0.015 \mathrm{Nm}$; (b) requested torque of $0.085 \mathrm{Nm}$.

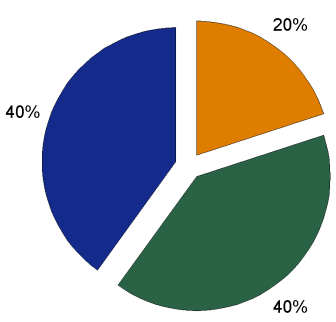

(a)

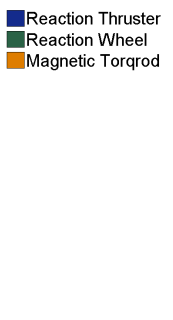

Reaction Thruster
Reaction Wheel
Magnetic Torqrod

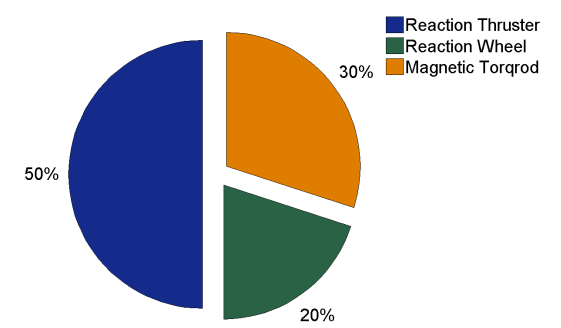

(b)

Figure 7. AMCM commanded torque: (a) requested torque of $0.015 \mathrm{Nm}$; (b) requested torque of $0.085 \mathrm{Nm}$.

Figure 8 presents the frequency of activation of each actuator throughout the simulation. The bars represent the frequency, in number of simulation steps, that a given commanded torque, in percentage, was sent to the actuator. Note that the most commanded torque vector was $30 \%, 50 \%$, and $20 \%$ for the reaction thruster, reaction wheel, and magnetic torqrod, respectively. It is worth to remember that this values represent the commanded torque which can be different from the applied one due to limitations and characteristics of the actuators. AMCM takes into account this constraints and selects the best solution even if the commanded torque is not applied.

The time angular response, which represents the time spent by each actuator to mitigate the angular error, is presented in figure 9. Four operations mode are compared: all-reaction thruster, all-reaction wheel, all-magnetic torqrod, and AMCM. The horizontal axis represents the time necessary to execute the attitude maneuver whereas the vertical axis represents the angular motion.

We can observe that although the magnetic torqrod provided the slowest response with the second highest overshoot, the steady state was even achieved. The reaction thruster's response was a little faster but also shown a overshoot and a disturbing behavior in the other axes due to its coupling to the rendezvous control system. Analyzing only the angular motion of the $Y_{B}$ axis, we can see that the response from AMCM and reaction wheel are very similar. In spite of AMCM has showed a short period of disturbance in $X_{B}$ and $Z_{B}$ axes, it has presented a satisfactory attitude response. The time translational response of the
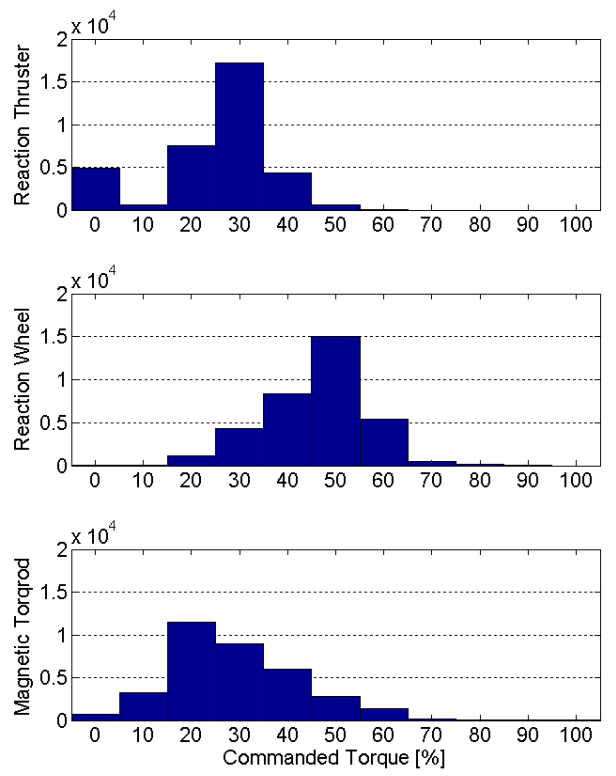

Figure 8. Frequency of activation of each actuator. 

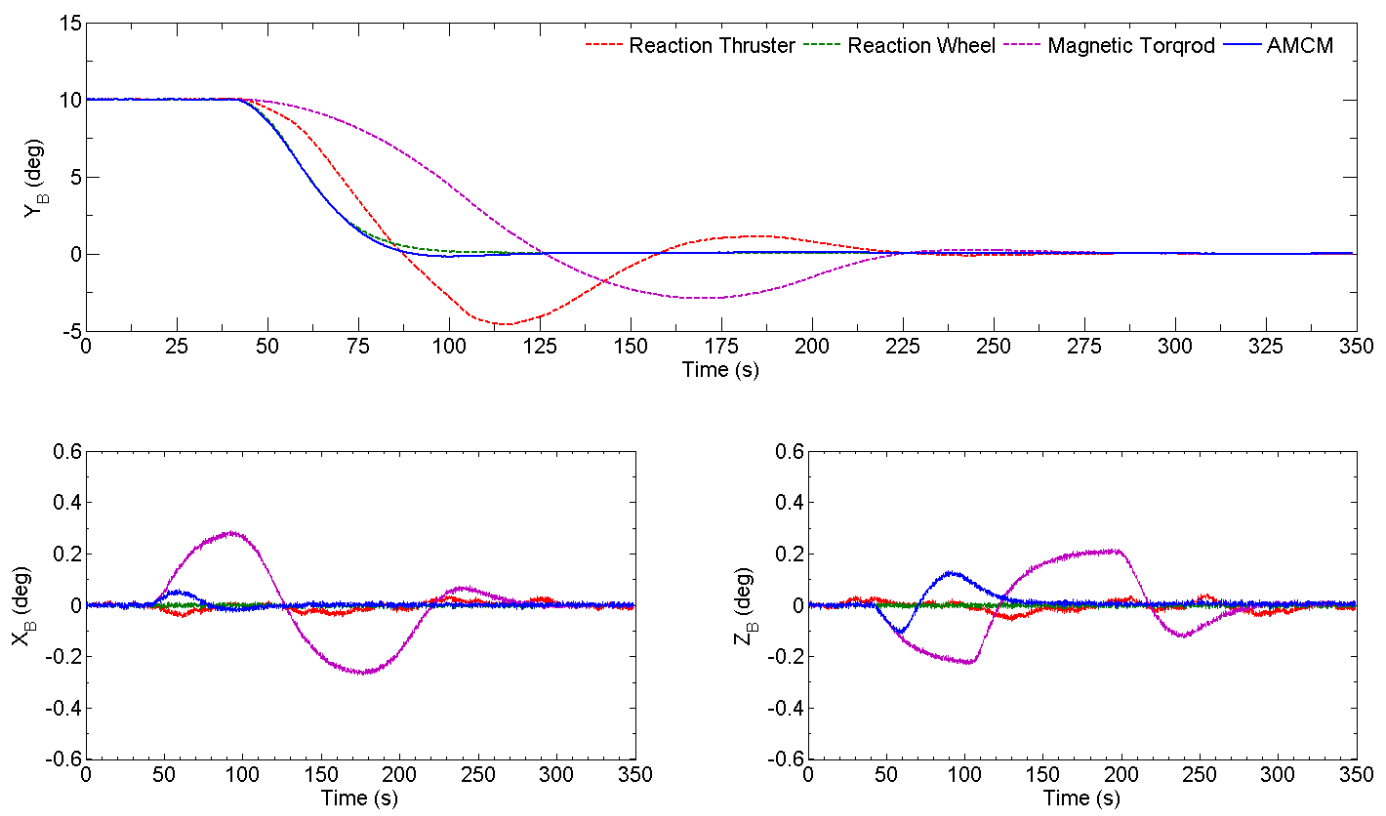

Figure 9. Time angular response of different operations mode.

final approach rendezvous using AMCM is shown in Figure 10. On the left side is presented the approaching motion through V-bar ( $X$ axis) whereas the motion under the remaining axes ( $Y$ and $Z$ ) are brought out on the right side of the figure. Three states are disclosed in this plot: the real state provided by the dynamics, the state measured by the sensors, and the state smoothed by the filter. It is important to point out the reduction of the measurement noise insofar as the chaser vehicle approaches the target vehicle.
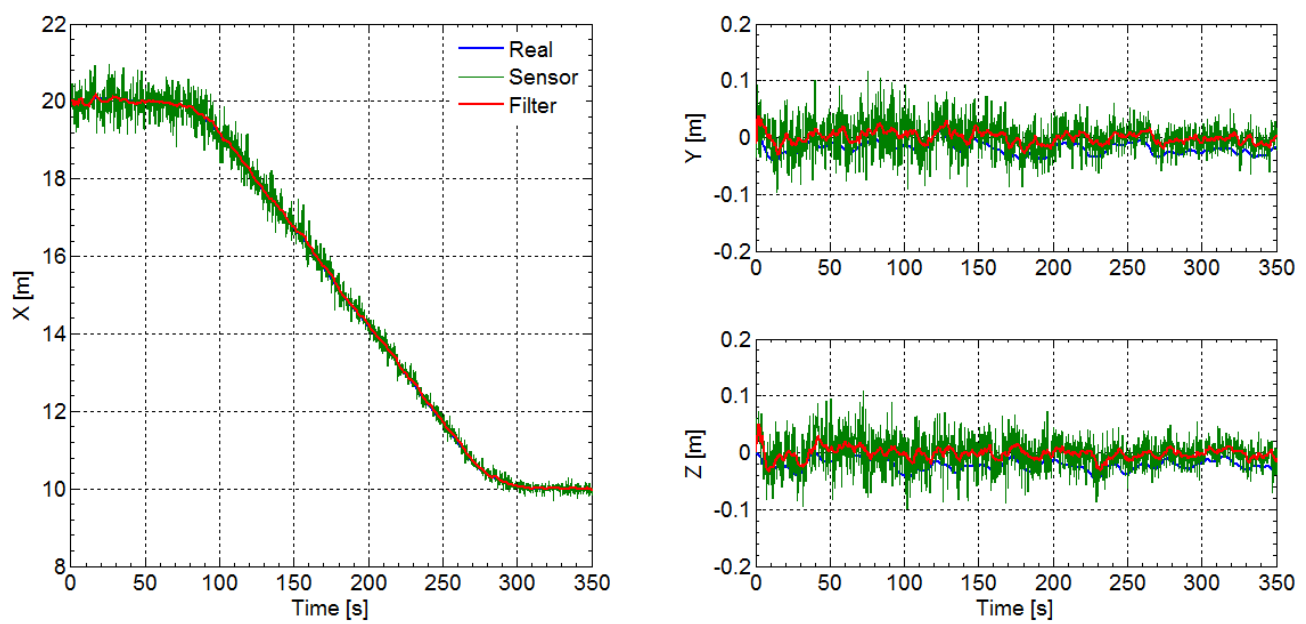

Figure 10. Time translational response of a spacecraft composed of actuators commanded by AMCM.

An outstanding way of analyzing the performance of a control system is through the visualization of the loop errors. Figure 11 shows four different types of translational position error: sensor / filter minus the real state (left side); and guidance minus real / filter (right side). The efficiency of the filter has been proved through the plots on the left side. All those plots have evidenced that the AMCM has worked properly in a coupled rendezvous and attitude control system.

In order to measure the efficiency of the proposed method over the GNC loop, some performance parameters have been analyzed, as shown in figure 12, such as: angular error integrated over time, fuel consumption, electrical charge consumption, and settling time. In fact, they are correlated with the objectives functions 

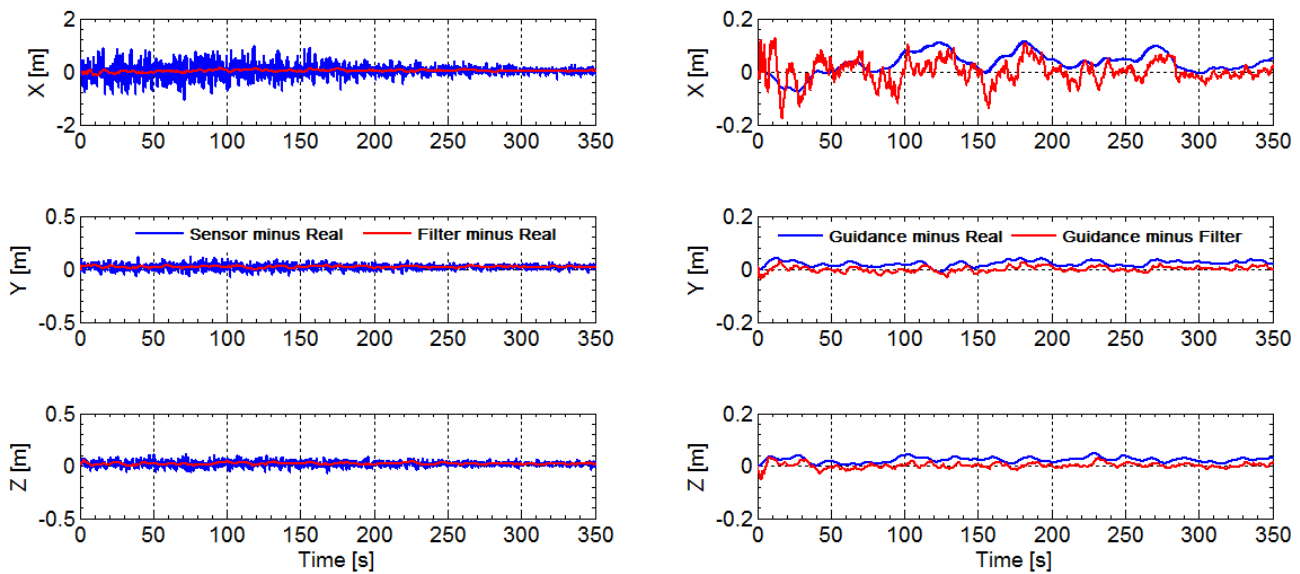

Figure 11. GNC loop errors including the real position (left) and the guidance position (right).

and represent another way to look through the performance. It is worth to mention that although the reaction wheel and magnetic torqrods are used only for attitude control, thrusters are always used to correct the deviation in the translational trajectory.

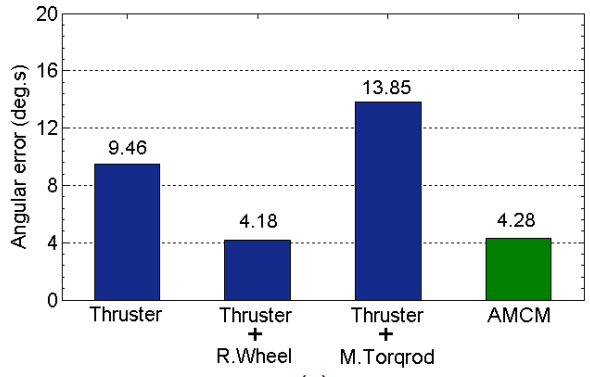

(a)

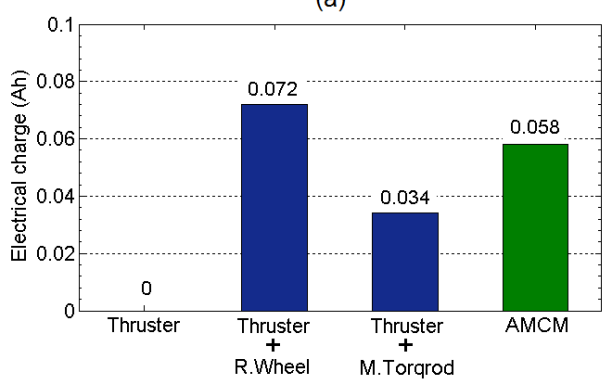

(c)

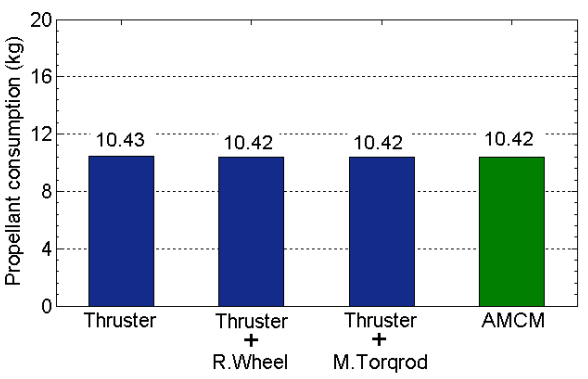

(b)

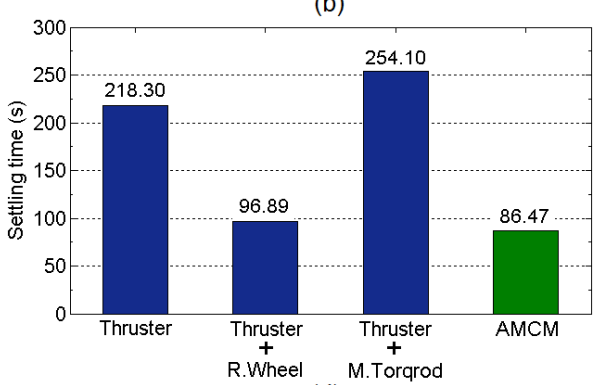

(d)

Figure 12. Performance parameters of the GNC loop: (a) angular error integrated over time; (b) fuel consumption; (c) electrical charge consumption; (d) settling time of the angular response.

Figure 12(a) presents the angular error of all axes integrated throughout the simulation for each actuator configuration. As expected, the magnetic torqrod had the highest value. The results provided by AMCM and reaction wheel were quite similar: 4.28 and 4.18 respectively. Concerning the propellant consumption shown in figure 12(b), the consumed amount of propellant of all configurations were quite similar. In addition, AMCM kept the same level of consumption of the all-thruster configuration. For obvious reasons, the electrical charge consumption, shown in figure 12(c), is null for the reaction thruster alternative and should not be taking into account in the comparison. The electrical signal to command the valve of the thruster has not been considered. The reaction wheel had a higher consumption than the magnetic torqrod whereas AMCM presented an intermediate result. The settling time of the time angular response is analyzed in figure 12(d). In fact, figure 12(a) and 12 (d) just quantify what was previously shown in figure 9 . The fastest response was achieve with AMCM $(88.47 \mathrm{~s})$ followed by the reaction wheel's response $(96.89 \mathrm{~s})$. 


\section{B. HIL Rendezvous Simulations}

In Section A, the multiobjective optimization approach has been validated through the comparison to different actuators configurations. The main difference, between the simulation of this section to that one presented before, is the utilization of a real visual camera sensor in a closed-loop control system instead of a mathematical model used before. Therefore, plots concerning the multiobjective optimization are not presented again due to the similarity of results. This section focuses on the results of the approaching and attitude maneuver and the capacity of the model for working in a real-time environment. The EPOS facility provides test and verification capabilities for the complete translational and rotational motion of two docking satellites. EPOS includes two industrial robots (separation ranging from $25 \mathrm{~m}$ to $0 \mathrm{~m}$ ) which are used for utmost realistic simulation of the real rendezvous and docking process (see figure 13).
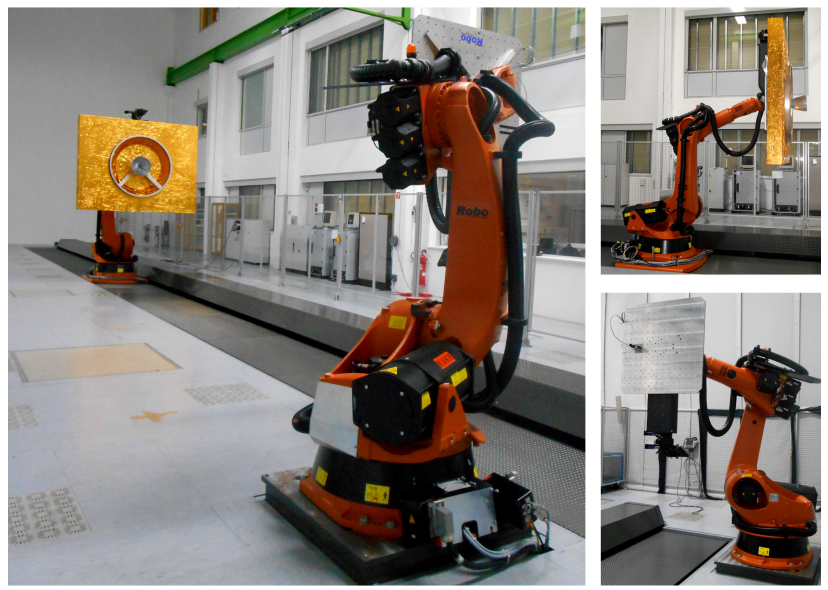

Figure 13. The robots of the European Proximity Operations Simulator (EPOS).

EPOS can be divided into the following three levels: the Local Robot Control (LRC) where all axes of the robots are separately controlled; the Facility Monitoring and Control system (FMC) where the entire facility is monitored and controlled in real-time with $250 \mathrm{~Hz}$ command rate, i.e., the real-time computers communicate with the robots sending commands every $4 \mathrm{~ms}$; and the Application Control System (ACS) where the actual RvD-simulation application is running. Ref. 14 provides additional information about EPOS. An overview about the EPOS set-up in closed-loop including the rendezvous and attitude simulator is presented in figure 14 .

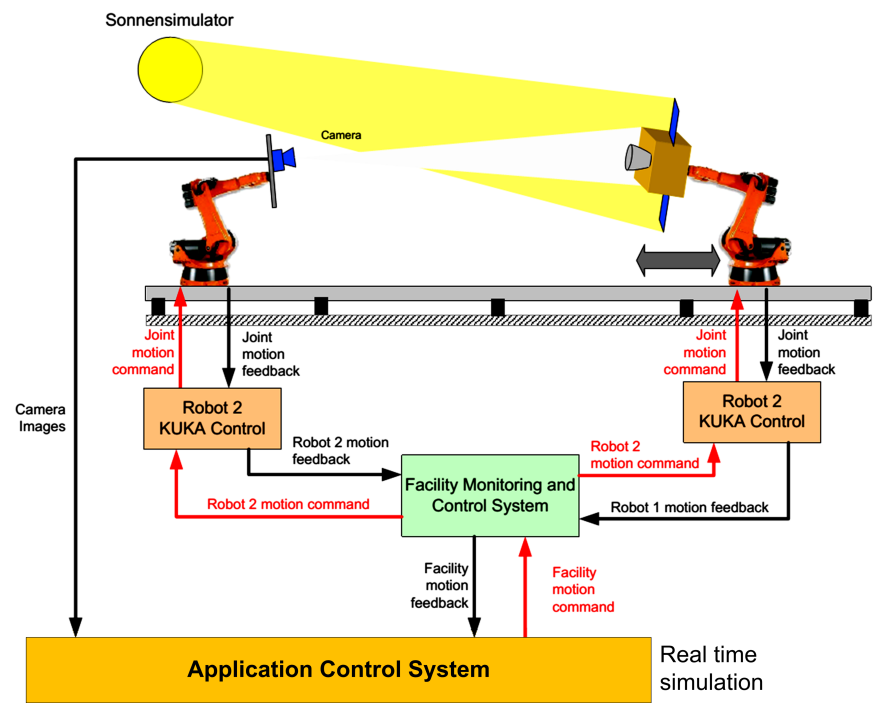

Figure 14. Set-up of EPOS in closed-loop control system. 
A complete closed-loop simulation of the entire rendezvous process is presented in this section. Here, the RCS is composed of thrusters with nominal thrust of $1 \mathrm{~N}$. AMCM is used to operate the actuators. The relative position and attitude is measured by a monochrome visual camera sensor (as specified in Table 1). The GNC loop, comprised of AMCM and actuators models, computes the new state of the satellite. Thereafter, the FMC computers convert the commanded state, expressed in CLW coordinates, to the global laboratory coordinate system before commanding it to the robots.

The first step of the closed-loop simulation is the evaluation of stability conditions at a hold point of $20 \mathrm{~m}$ from the target. Hence, an attitude maneuver to correct an initial angular error of 10 degrees is carried out. Afterward, an approaching maneuver through the V-bar axis is performed until the final hold point at $10 \mathrm{~m}$. Figure 15 presents the time translational (left) and angular (right) response of the complete simulation. As can be seen, the GNC loop was able to mitigate the state errors and to ensure stability conditions in all phases of the rendezvous scenario.
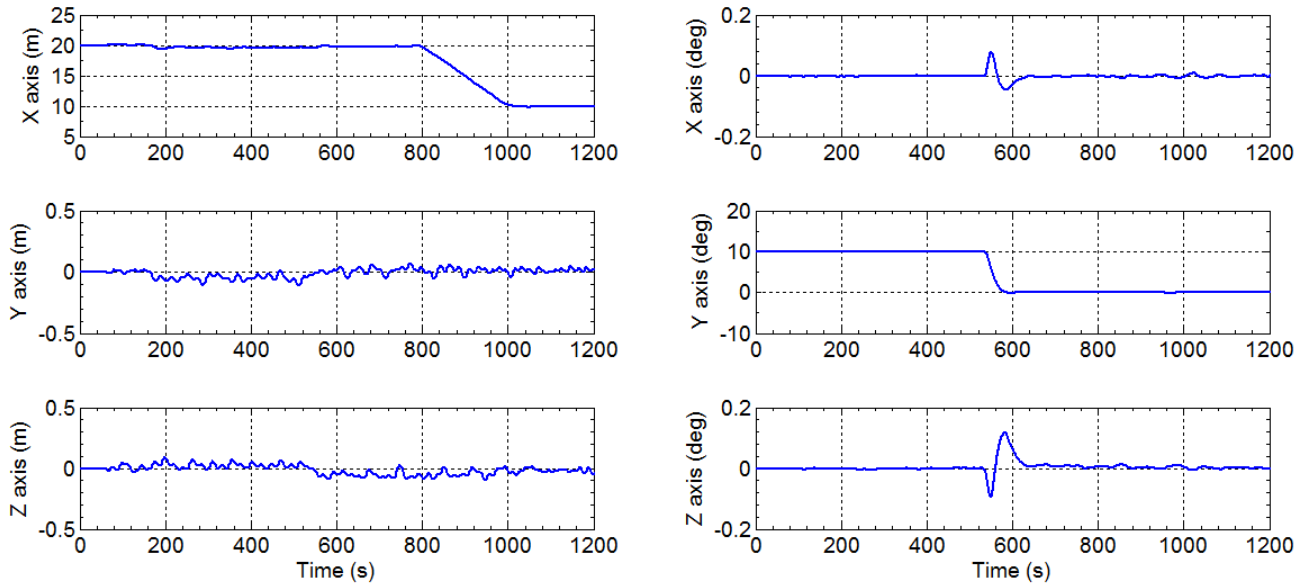

Figure 15. Time translational (left) and angular (right) response of the closed-loop simulation at EPOS.

Force and torque commands applied by the set of actuators are shown, in figure 16, for a given period of simulation (from 500s to 700s). On the left side of the plot is shown the force pulses applied by the RCS to correct the translational deviation whereas on the right side of the plot are presented the composition of torque commands applied by RCS, reaction wheels, and magnetic torqrods. AMCM worked properly commanding conflicting actuators in a real-time environment.
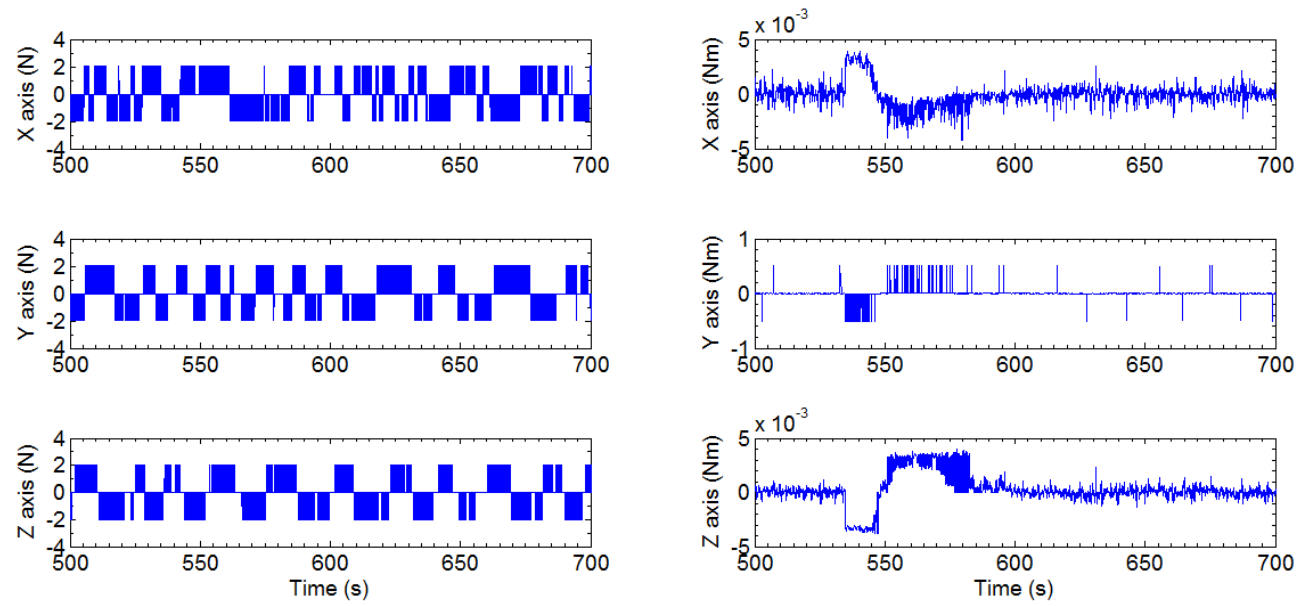

Figure 16. Applied force (left) and torque (right) commands as a function of time.

Figure 17 presents the relative position error between the state measured by the visual camera and the state commanded to the facility. A greater error can be seen at the initial steps. However, this deviation 
is reduced to low levels along the simulation. These plots prove that the proposed method, as well as the entire GNC loop, has worked properly in a real-time environment.
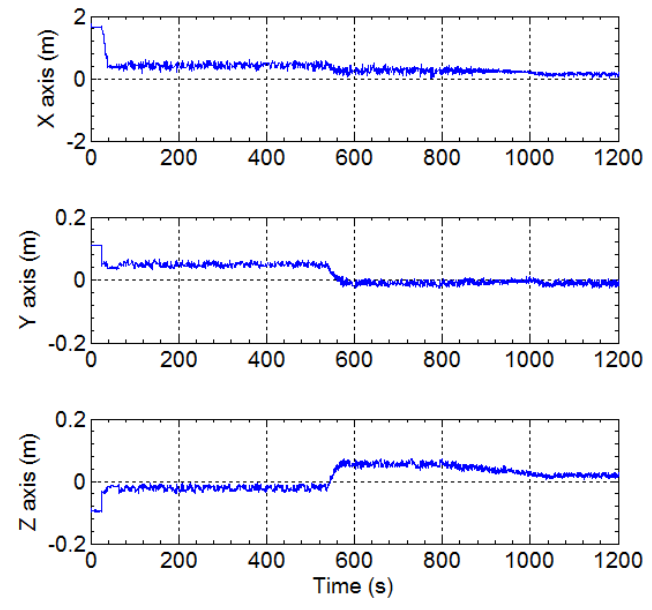

Figure 17. Relative position error of the visual camera.

\section{Conclusion}

The mixed actuator problem has been investigated in this paper and a novel approach to operate the spacecraft actuators has been proposed. The method solves, in real-time, a discrete multiobjective optimization problem at every control cycle. The problem is formulated such that the requested torque by the PID controller is quantized by discrete decision variables. The Smallest Loss Criterion provides the best compromise solution which optimizes simultaneously a group of four objective functions.

The proposed method is evaluated through both non-real-time and real-time simulations. It is not trivial to analyze the efficiency when we deal with conflicting objectives. However, simulations results of a coupled rendezvous and attitude maneuver have indicated that a good performance can be achieve with the proposed methodology AMCM. The method presented a satisfactory angular error, an accepted amount of fuel and electrical charge consumptions, with the fastest settling time when compared to the configuration of the same set of actuators. Tests of closed-loop rendezvous simulation have been successfully executed in a real-time system at EPOS facility of the German Aerospace Center. The stability conditions were ensured during the continuous approach phase as well as at the hold points. The state errors were kept within acceptable ranges. The implemented software demonstrated effectiveness and robustness proved to be able to generate reliable results. The work presented in this paper does not only solve an multiobjective optimization problem but it is also efficient in terms of a computational point of view.

\section{Acknowledgments}

This research was sponsored by CAPES Foundation, Ministry of Education of Brazil, process number BEX 3512/13-4. The authors also wish to thank the German Aerospace Center (DLR) and the National Institute for Space Research (INPE) which provided all the necessary support for the development of the work.

\section{References}

\footnotetext{
${ }^{1}$ Fehse, W., Automated Rendezvous and Docking of Spacecraft, Cambridge University Press, Cambridge, UK, 2003.

${ }^{2}$ Won, C., "Comparative Study of Various Control Methods for Attitude Control of a LEO Satellite," Aerospace Science and Technology, Vol. 3, No. 5, 1999, pp. 323-333.

${ }^{3}$ Hall, C. D., Panagiotis, T., and Shen, H., "Tracking Rigid Body Motion using Thrusters and Momentum Wheels," Journal of the Astronautical Sciences, Vol. 50, No. 3, Jul.-Sep. 2002, pp. 311-323.

${ }^{4}$ Roberts, B. A., et al., "Three-axis Attitude Control with Two Reaction Wheels and Magnetic Torquer Bars," AIAA
} 
Guidance, Navigation, and Control Conference and Exhibit, AIAA Paper 2004-5245, Aug. 2004.

${ }^{5}$ Bruno, D., "Contigency Mixed Actuator Controller Implementation for the Dawn Asteroid Rendezvous Spacecraft," AIAA SPACE 2012 Conference and Exposition, AIAA Paper 2012-5289, Sep. 2012.

${ }^{6}$ Macala, G. A., Lee, A. Y., and Wang, E. K., "Feasibility Study of Two Cassini Reaction Wheel/Thruster Hybrid Controllers," Journal of Spacecraft and Rockets, Vol. 51, No. 2, Mar.-Apr. 2014, pp. 574-585.

${ }^{7}$ Bals, J., Fichter, W., and Surauer, M., "Optimization of Magnetic Attitude- and Angular Momentum Control for Low Earth Orbit Satellites," Proceedings Third International Conference on Spacecraft Guidance, Navigation and Control Systems, SP-381, ESA, 1997, PP. 559-567.

${ }^{8}$ Fan, Z., Hua, S., Chundi, M., and Yuchang, L., "An Optimal Attitude Control of Small Satellite with Momentum Wheel and Magnetic Torqrods," Proceedings of the 4th World Congress on Intelligent Control and Automation, Vol. 2, IEEE, 2002, pp. 1395-1398.

${ }^{9}$ Zhang, X., and Zeng, M., "Multi-Objective Control of Spacecraft Attitude Maneuver Based on Takagi-Sugeno Fuzzy Model," Journal of Control Engineering and Applied Informatics, Vol. 14, No. 1, 2012, pp. 31-36.

${ }^{10}$ Marler, R. T., and Arora, J. S., "Survey of Multi-Objective Optimization Methods for Engineering," Structural and Multidisciplinary Optimization, Vol. 26, No. 6, 2004, pp. 369-395.

${ }^{11}$ Sullivan, B., and Akin, D., "A Survey of Serviceable Spacecraft Failures," AIAA Paper 2001-4540, Aug. 2001.

${ }^{12}$ Sellmaier, F., et al., "On-Orbit Servicing Missions: Challenges and Solutions for Spacecraft Opertaions," SpaceOps 2010 Conference, AIAA Paper 2010-2159, 2010.

${ }^{13}$ Arantes Jr., G., Rocco, E. M., Fonseca, I. M., Theil, S., "Far and Proximity Maneuvers of a Constellation of Service Satellites and Autonomous Pose Estimation of Customer Satellite Using Machine Vision," Acta Astronautica, Vol. 66, No. 9-10, 2010, pp. 1493-1505.

${ }^{14}$ Boge, T., Benninghoff, H., Zebenay, M., and Rems, F., "Using Robots for Advanced Rendezvous and Docking Simulation," SESP 2012: Simulation and EGSE facilities for Space Programmes, Noordwijk, The Netherlands, Sept. 2012.

${ }^{15} \mathrm{Ma}$, O., Flores-Abad, A., and Boge, T., "Use of Industrial Robots for Hardware-in-the-loop Simulation of Satellite Rendezvous and Docking," Acta Astronautica, Vol. 81, No. 1, 2012, pp. 335-347.

${ }^{16}$ Benninghoff, H., Rems, F., and Boge, T., "Development and hardware-in-the-loop test of a guidance, navigation and control system for on-orbit servicing," Acta Astronautica, Vol. 102, 2014, pp. 67-80.

${ }^{17}$ Wertz, J. R., Spacecraft Attitude Determination and Control, Astrophysics and Space Science Library, Dordrecht, Netherlands, 1978.

${ }^{18}$ Gomes dos Santos, W., Kuga, H. K., and Rocco, E. M., "Application of the Kalman Filter to Estimate the State of an Aerobraking Maneuver," Mathematical Problems in Engineering, Vol. 2013, 2013.

${ }^{19}$ Sidi, M. J., Spacecraft Dynamics $\&$ Control: A Practical Enginnering Approach, Cambridge Aerospace Series, Cambridge University Press, Cambridge, UK, 1997.

${ }^{20}$ Jazwinski, A. H., Stochastic Processes and Filtering Theory, Academic Press, New York, 1970.

${ }^{21}$ Boge, T., Benninghoff, H., and Tzschichholz, T., "Hardware-in-the-loop Rendezvous Simulation Using a Vision Based Sensor," 8th International ESA Conference on Guidance, Navigation and Control Systems, ESA, 2011.

22 Åström, K. J., Wittenmark, B., Computer-Controlled Systems: Theory and Design, 3rd ed., Prentice Hall, Beijing, China, 1997, Chap. 8.

${ }^{23}$ Johnson, M. A., and Moradi, M. H., PID Control: New Identification and Design Methods, Springer, London, 2005.

${ }^{24}$ Kristiansen, R., and Hagen, D., "Modelling of Actuator Dynamics for Spacecraft Attitude Control," Journal of Guidance, Control, and Dynamics, Vol. 32, No. 3, May-June 2009, pp. 1022-1025.

${ }^{25}$ Oliveira, T. C., Rocco, E. M., Ferreira, J. L., and Prado, A. F. B. A., "Minimum Fuel Low-Thrust Transfers for Satellites Using a Permanent Magnet Hall Thruster," Mathematical Problems in Engineering, Vol. 2013, 2013.

${ }^{26}$ Crawford, B. S., "Configuration Design and Efficient Operation of Redundant Multi-Jet Systems," Proceedings of AIAA Guidance Control and Flight Mechanics Conference, pp. 69-845, 1969.

${ }^{27}$ Press, W. H., Teukolsky, S. A., Vetterling, W. T., and Flannery, B. P., Numerical Recipes: The Art of Scientific Computing, 3rd ed., Cambridge University Press, Cambridge, England, UK, 2007.

${ }^{28}$ Sutton, G. P., and Biblarz, O., Rocket Propulsion Elements, 7th ed., John Wiley \& Sons, New York, 2001.

${ }^{29} \mathrm{Ge}$, S., and Cheng, H., "A Comparative Design of Satellite Attitude Control System with Reaction Wheel," First NASA/ESA Conference on Adaptive Hardware and Systems, IEEE, 2006, pp. 359-364.

${ }^{30}$ Arbinger, C., and Lübke-Ossenbeck, B., "Attitude Control," Handbook of Space Technology, edited by W. Ley, K. Wittmann and W. Hallmann, John Wiley and Sons, United Kingdom, 2009, pp. 332-361.

${ }^{31}$ Finlay, C. C., et al., "International Geomagnetic Reference Field: the Eleventh Generation," Geophysical Journal International, Vol. 183, No. 3, 2010, pp. 1216-1230.

${ }^{32}$ Silani, E., and Lovera, M., "Magnetic Spacecraft Attitude Control: A Survey and Some New Results," Control Engineering Practice, Vol. 13, No. 3, 2005, pp. 357-371.

${ }^{33}$ Cohon, J. L., Multiobjective Programming and Planning, Dover Publications, Mineola, NY, 2003.

${ }^{34}$ Ehrgott, M., and Gandibleux, X., Multiple Criteria Optimization: State of the Art Annotated Bibliographic Surveys, Kluwer Academic Publishers, New York, 2003, Chap. 8.

${ }^{35}$ Brualdi, R. A., Introductory Combinatorics, Pearson Education, New York, 1992.

${ }^{36}$ Pareto, V., Manuale di Economica Politica, Societa Editrice Libraria, Milan, translated into English by A. S. Schwier as Manual of Political Economy, edited by A. S. Schwier and A. N. Page, A.M. Kelley, New York, 1971.

${ }^{37}$ Rocco, E. M., Souza, M. L. O., and Prado, A. F. B. A., "Multi-objective Optimization Applied to Satellite Constellations I: Formulation of the Smallest Loss Criterion," Proceedings of the 54th International Astronautical Congress, IAC-03-A.3.01, IAC, Bremen, Germany, 2003. 
${ }^{38}$ Rocco, E. M., Souza, M. L. O., and Prado, A. F. B. A., "Further Application of the Smallest Loss Criterion in the Multi-objective Optimization of a Satellite Constellation," Proceedings of the 56th International Astronautical Congress, IAC, Fukuoka, Japan, 2005, pp. 3165-3172.

${ }^{39}$ Rocco, E. M., Souza, M. L. O., and Prado, A. F. B. A., "Station Keeping of Constellations Using Multiobjective Strategies," Mathematical Problems in Engineering, Vol. 2013, 2013.

${ }^{40}$ Schilling, D. A., Revelle, C., and Cohon, J., "An Approach to the Display and Analysis of Multiobjective Problems," Socio-Economic Planning Sciences, Vol. 17, No. 2, 1983, pp. 57-63. 